TECHNICAL NOTE

\author{
S. Toyota \\ K. Iwaisako \\ H. Takimoto \\ T. Yoshimine
}

\title{
Intravenous 3D Digital Subtraction Angiography in the Diagnosis of Unruptured Intracranial Aneurysms
}

SUMMARY: Recent progress in digital subtraction angiography (DSA) devices makes it possible to perform rotational angiography with high resolution and high sensitivity. We tried intravenous (IV) 3D DSA in patients who had undergone MR angiography (MRA) suggestive of unruptured intracranial aneurysms. IV 3D DSA can be used as an alternative method for imaging unruptured intracranial aneurysms suggested on MRA.

C erebral angiography is an essential technique in the diagnostic imaging and preoperative evaluation of cerebral aneurysm; however, it is invasive and carries a certain risk of complications. ${ }^{1}$ Intravenous (IV) digital subtraction angiography (DSA) has been used in some patients for the less invasive examination of cerebral, abdominal, and peripheral arteries. ${ }^{2,3}$ With the recent progress in DSA devices, it is possible to perform rotational angiography with high resolution and high sensitivity. ${ }^{4,5}$ We performed IV 3D DSA for the diagnosis of unruptured intracranial aneurysms.

\section{Technique and Representative Cases}

From August 2006 to December 2006, IV 3D DSA was performed in 29 outpatients ( 10 men, 19 women; mean age, $58.8 \pm 12.4$ years) who had undergone time-of-flight MR angiography (MRA) suggestive of unruptured intracranial aneurysms. With IV 3D DSA, intracranial aneurysms were recognized in 11 patients (internal carotid artery, 7 patients; anterior communicating artery, 1 patient; middle cerebral artery, 1 patient; basilar artery, 1 patient; vertebral artery, 1 patient), but not in 18 patients.

\section{Description of the Technique of IV 3D DSA}

IV 3D DSA was obtained with rotational angiographies performed on a C-Arm (Infinix Celeve VS; Toshiba, Tokyo, Japan). This covers a total angular range of $200^{\circ}$, with a first rotation of $40 \%$ s to acquire the mask images, a second rotation to return to the starting position, and a third rotation of $40 \% \mathrm{~s}$ to acquire the opacified images. The type of detector in this system was an image intensifier (RTP12303J-G9E; Toshiba).

An 18-gauge angiocatheter was inserted into the right antecubital vein and linked with a connective tube for power injection. First, 15 $\mathrm{mL}$ of nonionic contrast medium (Omnipaque 350; Daiichi Seiyaku, Tokyo, Japan) was injected with a pump at a rate of $9 \mathrm{~mL} / \mathrm{s}$. The delay time from the start of contrast injection to the time when the common carotid arteries were filled with contrast was measured with DSA. Then $50 \mathrm{~mL}$ of the contrast medium was injected at a rate of $9 \mathrm{~mL} / \mathrm{s}$. The acquisition of source images was started after the start of contrast injection. The acquisition matrix was $512 \times 512$.

This protocol resulted in a rotational series of 200 subtracted im-

Received April 14, 2007; accepted after revision June 22

From the Department of Neurosurgery (S.T., K.I., H.T.), Osaka Neurological Institute, Osaka, Japan; and the Department of Neurosurgery (T.Y.), Osaka University Medical School, Osaka, Japan.

Please address correspondence to Shingo Toyota, MD, PhD, Department of Neurosurgery, Osaka Neurological Institute, 2-6-23 Shounai, Takara-machi, Toyonaka, Osaka 561-0836, Japan; e-mail: stshto@aol.com

DOI 10.3174/ajnr.A0822 ages. Rotational angiography data were automatically transferred to a personal computer (XIDF-100A; Toshiba), which reconstructed 3D volume data. The matrix of transferred data were transformed from $512 \times 512$ to $256 \times 256$ by voxel addition. The $3 \mathrm{D}$ volume data were transferred to a commercially available multimodality $3 \mathrm{D}$ workstation (ZIO M900TXA; ZIO Software, Tokyo, Japan), processed, and presented. Available visualization of algorithms included maximum intensity projection (MIP) and volume rendering.

\section{Representative Cases}

Case 1: A 72-year-old woman. MRA was suggestive of an aneurysm in the right internal carotid artery (Fig $1 A$ ). With volume rendering images of IV 3D DSA, the aneurysm and its relationship with the surrounding arterial branches were depicted (Fig 1B). The size of the aneurysm could be measured with MIP (Fig 1C). She underwent additional intra-arterial (IA) 3D DSA due to a proposal of surgical treatment. IA 3D DSA was performed with the injection of $15 \mathrm{~mL}$ of nonionic contrast medium (Iopamiron 300; Nihon Schering, Osaka, Japan) into the right common carotid artery at a rate of $3 \mathrm{~mL} / \mathrm{s}$. With volume rendering images of IA 3D DSA, the aneurysm was depicted in higher spatial resolution, and small vessels surrounding the aneurysm were revealed (Fig $1 D$ ).

Case 2: A 73-year-old man. MRA was suggestive of a small aneurysm in the anterior communicating artery (Fig $2 A)$. With volume rendering images of IV 3D DSA, a small aneurysm was recognized and the surrounding arterial branches were depicted (Fig $2 B$ ). The aneurysm measured with MIP (Fig $2 C$ ) was so small that additional IA DSA was not performed.

Case 3: A 67-year-old woman. MRA was suggestive of a small aneurysm in the left middle cerebral artery (Fig $3 A$ ), but it was not recognized with volume rendering images or MIP of IV 3D DSA (Fig $3 B,-C)$. The suggested lesion was thought to be the curved portion of the M1 segment of the left middle cerebral artery.

Case 4: A 45-year-old man. MRA was suggestive of a small aneurysm in the anterior communicating artery (Fig 4A), but it was not recognized with volume rendering images or MIP of IV 3D DSA (Fig $4 B,-C)$. The suggested lesion was thought to be the curved portion of the anterior communicating artery.

\section{Discussion}

The major advantage of IV DSA is the ability to obtain arteriograms relatively noninvasively through an IV injection of contrast medium, hence reducing the risk and cost of standard arterial catheterization. IV DSA has been used in some cases for the less invasive examination of cerebral, abdominal, and peripheral arteries $^{3}$; however, because these vessels are not ad- 

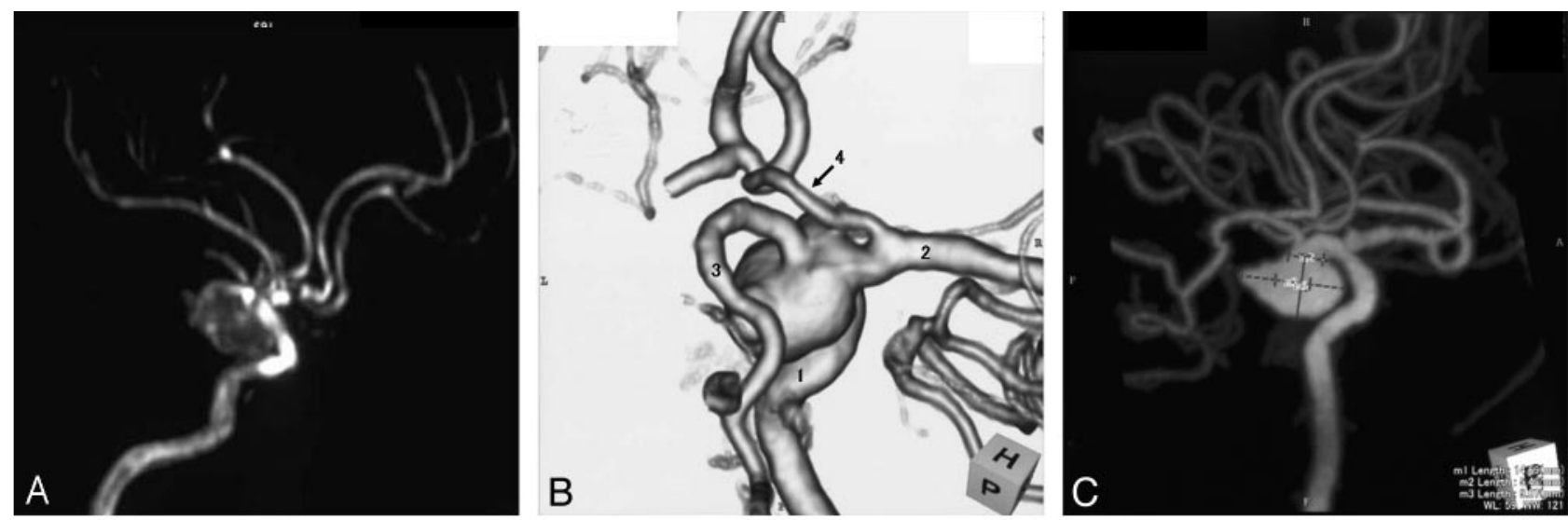

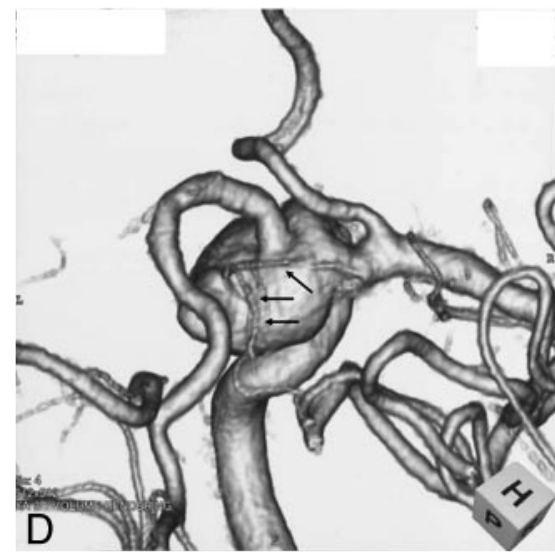

equately demonstrated in all cases, intra-arterial DSA is mainly being performed. ${ }^{2}$ Because of progress in high-speed rotation of the $\mathrm{C}$-arm and the technique of high-speed acquisition of images, rotational angiography can be performed with a high resolution. ${ }^{4,5}$ The spatial resolution and sensitivity of 3D images of our system are excellent because it can obtain 200 projection images in 1 acquisition and because the image intensifier has a high conversion factor $\left(40\left\{\mathrm{~cd} / \mathrm{m}^{2}\right\} /\{\mu \mathrm{G} \mathrm{y} / \mathrm{s}\}\right)$ and high contrast ratio (30:1 at $10 \%$ area contrast). These developments in DSA devices make IV 3D DSA possible. By manipulation of the 3D DSA data, lesions suggested on MRA can be depicted precisely in various ways. Volume rendering images of IV 3D DSA were more effective in demonstrating the relationship between the aneurysm and the adjacent vessels
Fig 1. MRA time-of-flight image (case 1). $A$, An aneurysm in the right internal carotid artery was suggested. $B$, Volume rendering image of IV 3D DSA (case 1). The aneurysm and its relationship with the surrounding arterial branches were anterior cerebral artery. C, MIP of IV 3D DSA (case 1). The size of the aneurysm was $14.7 \times 9.1 \mathrm{~mm}$ for the aneurysm sack and $5.5 \mathrm{~mm}$ for the neck. D, Volume rendering images of IA 3D DSA (case 1). The aneurysm was depicted in higher spatial resolution, and small vessels surrounding the aneurysm were revealed (arrows).

than MIP imaging. ${ }^{6}$ For examination of the existence of small aneurysms and for analysis of the size of aneurysms and vessels, MIP produced from the original nonthreshold 3D data was feasible. ${ }^{6}$

When data were transferred to the personal computer for reconstruction, the matrix size was transformed from $512 \times$ 512 to $256 \times 256$ by voxel addition. This procedure is necessary for better contrast resolution because the contrast medium is diluted by a much larger volume of blood in IV 3D DSA. ${ }^{2}$ Therefore, compared with IA 3D DSA, the spatial resolution of IV 3D DSA is inferior, especially in the demonstration of small vessels; however, we think that IV 3D DSA has enough quality, at least as "the second check" of unruptured intracranial aneurysms suggested on MRA, even though small perforators cannot be depicted precisely. In our series, only 3 patients needed to undergo IA 3D DSA additionally due to a proposal for surgical treatment. As information about surgical treatment, IV 3D DSA was not inferior to IA 3D DSA in all 3 patients.

In the field of neurosurgery, 3D CTA is the most available method for the IV evaluation of cerebrovascular lesions. ${ }^{7}$ Recent studies have suggested that 3 D CTA is equal or superior to
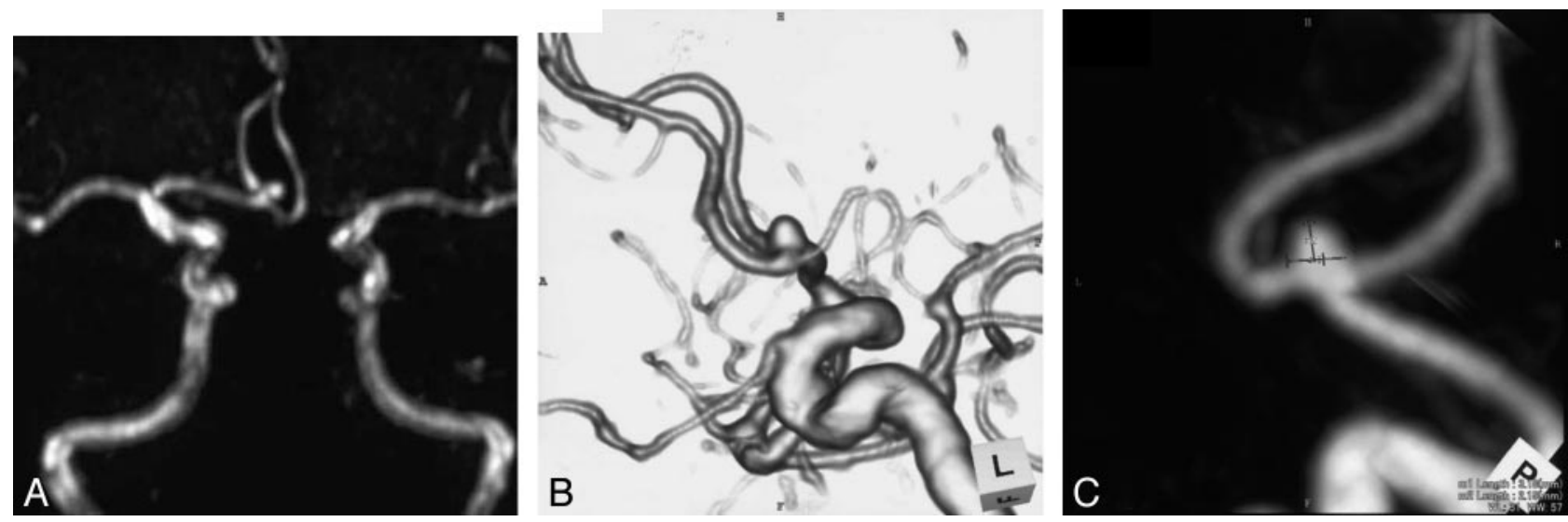

Fig 2. MRA time-of-flight image (case 2). A, A small aneurysm in the anterior communicating artery was suggested. B, Volume rendering images of IV 3D DSA (case 2). A small aneurysm was recognized, and the surrounding arterial branches were depicted. C, MIP of IV 3D DSA (case 2). The size of the aneurysm was $3.2 \times 2.2 \mathrm{~mm}$ for the aneurysm sack and $3.2 \mathrm{~mm}$ for the neck. 

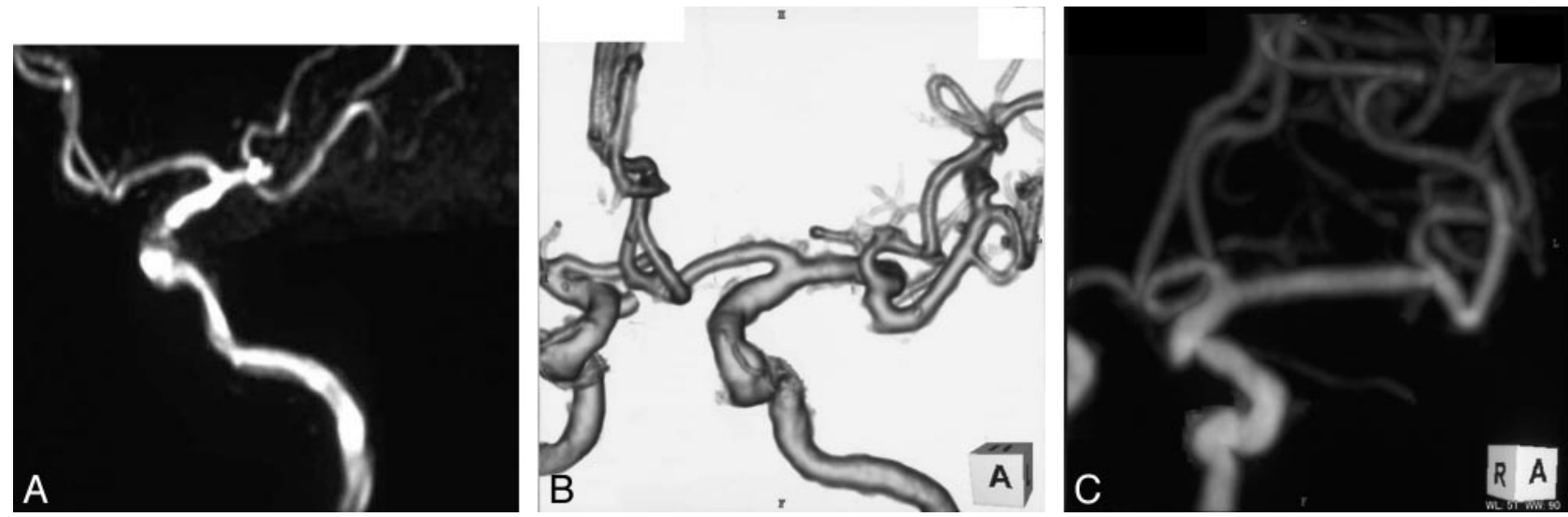

Fig 3. MRA time-of-flight image (case 3). A, A small aneurysm in the left middle cerebral artery was suggested. $B$, Volume rendering images of IV 3D DSA (case 3). The suggested aneurysm was not recognized. The suggested lesion was thought to be the curved portion of the M1 segment of the left middle cerebral artery. C, MIP of IV 3D DSA (case 3). The suggested aneurysm was not recognized.
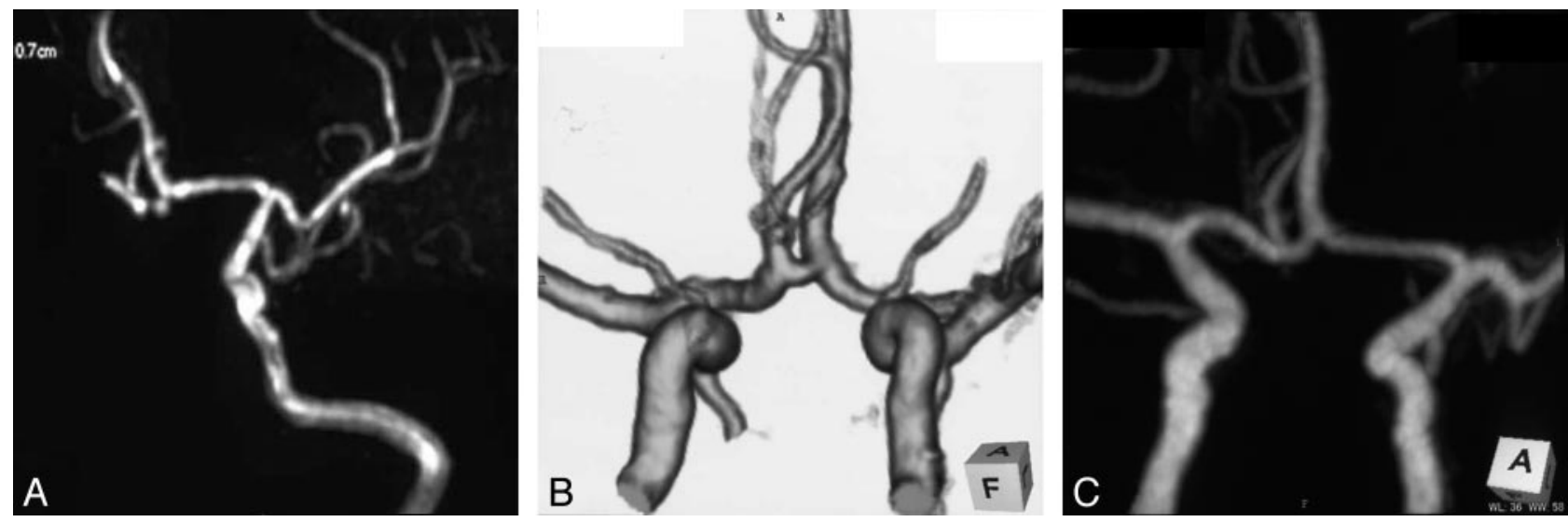

Fig 4. MRA time-of-flight image (case 4). $A, A$ small aneurysm in the anterior communicating artery was suggested. B, Volume rendering images of IV 3D DSA (case 4). The suggested aneurysm was not recognized. The suggested lesion was thought to be the curved portion of the anterior communicating artery. $C$, MIP of IV 3D DSA (case 4). The suggested aneurysm was not recognized.

conventional angiography in characterizing aneurysms for surgical planning. ${ }^{8}$ Neurosurgeons can use 3D CTA more easily than IV 3D DSA because they are accustomed to using a helical CT scanner that can perform multipurposely. ${ }^{7}$ Despite some disadvantages, we think that IV 3D DSA may be more suitable for preoperative study of endovascular treatment than 3D CTA, because the question of whether a working view for endovascular treatment is reproduced on the C-arm can be checked with IV 3D DSA. $^{6}$

IV 3D DSA can be performed more quickly and safely than conventional angiography, especially in outpatients, without the risk and cost of arterial catheterization. IV DSA is generally a very safe procedure with low morbidity, ${ }^{2}$ but there have been a few descriptions of the extravasation of contrast material during IV DSA because of its high rate of injection. ${ }^{9}$ This complication occurs in approximately $5.2 \%$, with an infusion rate of $14 \mathrm{~mL} / \mathrm{s} .{ }^{10} \mathrm{In}$ our series, the rate was adjusted to $9 \mathrm{~mL} / \mathrm{s}$, which was less than in Pinto et $\mathrm{al}^{10}$, and we did not experience extravasation of contrast material during IV 3D DSA, or other complications.

\section{Conclusion}

With the recent progress in DSA devices, we could perform IV 3D DSA less invasively in the field of neuroradiology. Despite some disadvantages, IV 3D DSA can be used as an alternative method for imaging unruptured intracranial aneurysms suspicious on MRA.

\section{References}

1. Grzyska U, Freitag J, Zeumer H. Selective cerebral intraarterial DSA: complication rate and control of risk factors. Neuroradiology 1990;32:296-99

2. Butler $\mathrm{P}$. Intravenous digital subtraction angiography. $\mathrm{Br} J \mathrm{Hosp} \mathrm{Med}$ 1986;35:30-36

3. Modic MT, Weinstein MA, Pavlicek W, et al. Intravenous digital subtraction angiography: peripheral versus central injection of contrast material. Radiology 1983;147:711-15

4. Tu RK, Cohen WA, Maravilla KR, et al. Digital subtraction rotational angiography for aneurysms of the intracranial anterior circulation: injection method and optimization. AJNR Am J Neuroradiol 1996;17:1127-36

5. Abe T, Hirohata M, Tanaka N, et al. Clinical benefits of rotational 3D angiog raphy in endovascular treatment of ruptured cerebral aneurysm. AJNR Am J Neuroradiol 2002;23:686-88

6. Missler U, Hundt C, Wiesmann M, et al. Three-dimensional reconstructed rotational digital subtraction angiography in planning treatment of intracranial aneurysms. Eur Radiol 2000;10:564-68

7. Tacke J, Klein HM, Bertalanffy $\mathrm{H}$, et al. Clinical significance of three-dimensional helical CT in neurosurgery. Minim Invasive Neurosurg 1997;40:30-35

8. Korogi Y, Takahashi M, Katada K, et al. Intracranial aneurysms: detection with three-dimensional CT angiography with volume rendering - comparison with conventional angiographic and surgical findings. Radiology 1999;211:497-506

9. Ball JB Jr, Lukin RR, Tomsick TA, et al. Complications of intravenous digital subtraction angiography. Arch Neurol 1985;42:969-72

10. Pinto RS, Manuell M, Kricheff II. Complications of digital intravenous angiography: experience in 2488 cervicocranial examinations. AJR Am J Roentgenol 1984;143:1295-99 